EPJ Web of Conferences 47, 02001 (2013)

DOI: $10.1051 /$ epjconf/20134702001

(C) Owned by the authors, published by EDP Sciences, 2013

\title{
The SOAPS project - Spin-orbit alignment of planetary systems
}

\section{Exoplanets' evolution histories in systems with different architectures}

\author{
F. Faedi ${ }^{1, a}$, Y. Gómez Maqueo Chew ${ }^{1,2}$, L. Fossati $^{3}$, D. Pollacco ${ }^{1}$, \\ A. McQuillan ${ }^{4}$, L. Hebb ${ }^{5}$, W.J. Chaplin ${ }^{6}$ and S. Aigrain ${ }^{4}$ \\ ${ }^{1}$ Department of Physics, University of Warwick, Coventry CV4 7AL, UK \\ 2 Physics and Astronomy Department, Vanderbilt University, Nashville, Tennessee, USA \\ 3 Argelander-Institut für Astronomie der Universität Bonn, Auf dem Hügel 71, 53121 Bonn, \\ Germany \\ 4 Oxford Astrophysics, Keble Road, Oxford OX1 3RH, UK \\ 5 University of Washington, Department of Astronomy, Box 351580, Seattle, WA 98195, USA \\ 6 School of Physics and Astronomy, University of Birmingham, Edgbaston, Birmingham \\ B15 2TT, UK
}

\begin{abstract}
The wealth of information rendered by Kepler planets and planet candidates is indispensable for statistically significant studies of distinct planet populations, in both single and multiple systems. Empirical evidences suggest that Kepler's planet population shows different physical properties as compared to the bulk of known exoplanets. The SOAPS project, aims to shed light on Kepler's planets formation, their migration and architecture. By measuring $v \sin i$ accurately for Kepler hosts with rotation periods measured from their high-precision light curves, we will assess the alignment of the planetary orbit with respect to the stellar spin axis. This degree of alignment traces the formation history and evolution of the planetary systems, and thus, allows to distinguish between different proposed migration theories. SOAPS will increase by a factor of 2 the number of spin-orbit alignment measurements pushing the parameters space down to the SuperEarth domain. Here we present our preliminary results.
\end{abstract}

\section{INTRODUCTION}

After the discovery of the first Hot Jupiter [1], several scenarios have been proposed to explain the migration of planets into the observed close-in orbits, including disc migration, planet-planet scattering, and Kozai perturbations. The true obliquity between the planet orbit and the stellar spin axis, is a current observable that can distinguish between the different migration scenarios. For example, classical disc migration theory (e.g., [2]) suggest that the tidal interaction between the planets and the planetary disc yields aligned systems, where the stellar spin axis is perpendicular to the orbital plane. Other scenarios, like planet-planet scattering (e.g., $[3,4]$ ), and perturbations induced by a distant, more massive object (Kozai effect,[5]), predict spin-orbit misalignment. The currently available information about the planetary spin-orbit alignment mostly comes from measurements of the Rossiter-McLauglin (RM) effect $[6,7]$, or $\lambda$, the sky-projection of the true spin-orbit alignment $\psi$ (see Fig. 1 from [8]; and also

\footnotetext{
ae-mail: f.faedi@warwick.ac.uk
} 

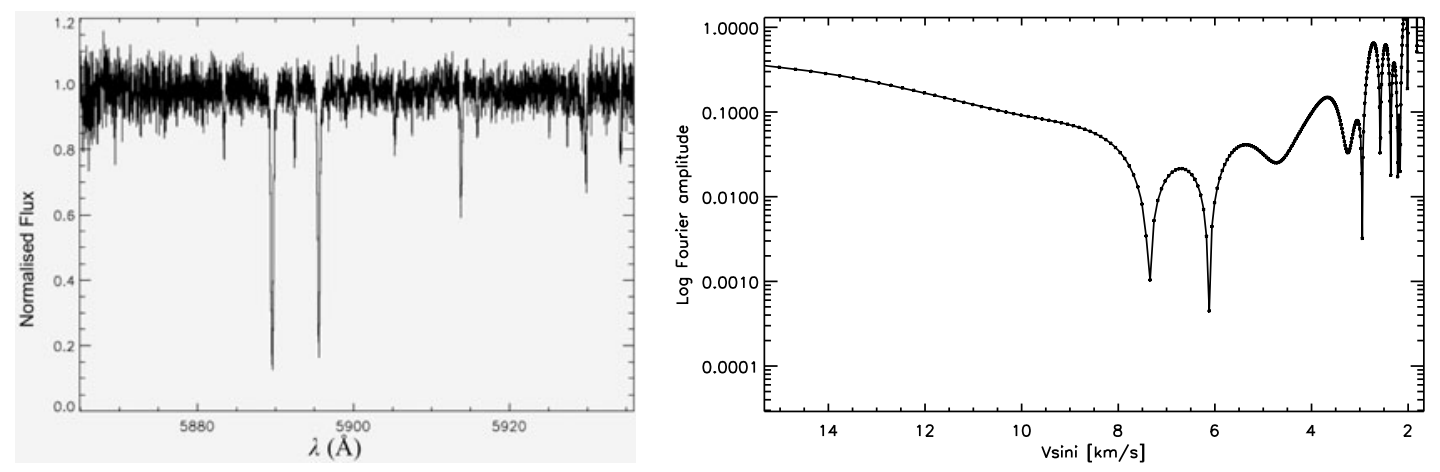

Figure 1. Left-panel shows an extract of the Subaru/HDS spectrum for Object-1 after the continuum normalisation. The spectrum was obtained with an exposure of 780s and a peak S/N of 24 at $\lambda=5700 \AA$. Right-panel shows the Fourier transform of the LSD profile for Object-1. We assumed a rigidly rotating star and a linear limb darkening model. See text for more details.

$[9,10])$ and show that $\sim 40 \%$ of the systems are substantially misaligned challenging the previously favored disc migration theory. Winn et al. [11] suggest that a possible correlation between misalignment and stellar convective turn-over time exists. Given a few exceptions, only hot stars $\left(T_{\text {eff }}>6250 \mathrm{~K}\right)$ with thin convective zones, are misaligned because tidal dissipation causes cooler hosts with larger outer convection zones to realign. The misaligned cool stars are those with the weakest tidal effects (i.e., longest P). Independently, [12] found that more massive stars $\left(>1.2 M_{\odot}\right)$ harbouring transiting exoplanets have estimated $v \sin i$ 's smaller than expected for a given age, as compared to a large sample of random field stars from [13]), supporting Winn's hypothesis (see Fig. 4 by [12]). Despite the numerous discoveries, Kepler transiting systems are too small ( $>76 \%$ are smaller than Neptune [14]), and their host stars are too faint and too slowly rotating to allow measurements of their RM effects. Thus, only by comparing the stellar spin axis, $I_{\star}$, to the planets orbital inclinations (via $v \sin i$ and $\mathrm{P}_{\text {rot }}$ measurements), we can probe their spin-orbit alignments.

In Sect. 2 we describe the analysis method and our observations. In Sect. 3 we present some preliminary result in Sect. 3 .

\section{THE SOAPS PROJECT}

With the SOAPS project we will investigate, for the first time, planetary architectures and compare the evolution histories of: (1) single planet systems to multi-planet systems; (2) hot Jupiters to cooler Jupiters; and (3) gas giants to rocky planets. For any star with accurate measurements of $v \sin i$ and rotation period $\left(\mathrm{P}_{r o t}\right)$, the stellar inclination $\left(I_{\star}\right)$ in the line of sight is determined as per $\sin I_{\star}=$ $v \sin i \mathrm{P}_{r o t} / 2 \pi R_{\star}$. In addition, in transiting systems the orientation of the planetary orbital plane is known. Thus, transiting systems for which the stellar $\mathrm{P}_{\text {rot }}$ and $v \sin i$ are measured, allow their spin-orbit alignment to be examined. We note that a similar study has been done by [8]. However, our approach and method of analysis are significantly different and explained below.

\subsection{Sample selection and observations}

We obtained our Kepler targets from the 1235 Kepler candidates of [14] and we selected only objects for which we measured, reliably, rotation periods from the Kepler first data quarter. The light curves were analysed using the method from [15]. Targets were selected by magnitude $(\mathrm{V}<14)$, spectral type $\left(5500<T_{\text {eff }}<7100 \mathrm{~K}\right)$ in the range to straddle the boundary identified by $[11,12]$, and also by planet multiplicity, in order to maximise the parameter space sampled. The resulting sample is composed of 


\section{Hot Planets and Cool Stars}

about 80 objects however, with the new Kepler release [16], and more available Kepler data, our sample will increase. Our sample includes slow and fast rotators, however below we describe the sample of slow rotators; observations for the sample of fast rotators are ongoing. We observed with the Subaru 8-m telescope and the high resolution echelle spectrograph HDS. With 1 night of HDS time (July 2012) we observed about 40 targets and 15 standard stars (slow rotators) with spectral types similar to that of the planet host in order to calibrate the shape of the intrinsic profile, plus instrumental broadening from their least-square deconvolution (LSD) profile, as described below (Sect. 2.2). We aimed to reach a peak S/N of $\sim 20$ with a $0.2^{\prime \prime}$ slit, and the standard setup Yc, centered at $\sim 5700 \AA$, with a spectral resolution of $\mathrm{R}=160000$. An extract of a HDS spectrum is shown in Fig. 1-left panel. The median Kepler magnitude of our targets is $\mathrm{V}=13.5$, with a typical exposure time of $\sim 800 \mathrm{~s}$. The spectral resolution is the major limiting factor in determining the expected low $v \sin i$ values of our targets $\left(2.0<v \sin i<6.0 \mathrm{~km} \mathrm{~s}^{-1}\right)$. With the selected instrumental setting we were able to measure, reliably, $v \sin i$ as low as $2.0 \pm 0.3 \mathrm{~km} \mathrm{~s}^{-1}$, and even lower. The HDS data (including calibrations) have been reduced using $\operatorname{IRAF}^{1}$ and the Subaru data reduction tools.

\subsection{Analysis method}

We measure $v \sin i$ using the Fourier transform methods (e.g., [17] to distinguish between the rotation and macro-turbulence $\left(v_{\text {mac }}\right)$ contributions. Each spectral line is a convolution of various overlapping profiles: intrinsic line profile (determined by stellar and atomic parameters), plus instrumental, rotational and $v_{\mathrm{mac}}$ broadening. With the Fourier technique $v \sin i$ and $v_{\mathrm{mac}}$ can be independently extracted. Assuming a rigidly rotating star and linear limb darkening, the zeros of the Fourier transformed line profile scales with $v \sin i$ [18], while the side lobes structure provides information on $v_{\text {mac }}$, albeit at high enough $\mathrm{S} / \mathrm{N}$. Because the majority of Keper transiting planet host-stars is far too faint to obtain high resolution spectra with the necessary high $\mathrm{S} / \mathrm{N}$, we will extract an overall high $\mathrm{S} / \mathrm{N}$ line profile for each star applying the LSD technique [19]. We will then derive the shape of the intrinsic profile, plus instrumental broadening, in two different ways: i) from the LSD profile of very slowly rotating stars with similar spectral type (also included in our HDS target list); ii) from the LSD profile of synthetic spectra, calculated with the appropriate stellar parameters, given in the Kepler input catalog [20], and broadened with the HDS spectral resolution. Finally, we will use LSD profiles calculated from synthetic spectra broadened with various combinations of $v \sin i$ and $v_{\text {mac }}$ (treated as radial-tangential) values to $v \sin i$ and $v_{\text {mac }}$, in the Fourier space, with a similar approach to that of [21].

\section{PRELIMINARY RESULTS}

Here we present our very preliminary results for two targets in our sample. Object-1 is a F5-F6 star with a temperature of $T_{\text {eff }} \sim 6130 \mathrm{~K}$ hosting a SuperEarth with an orbital period of about 2.8 days. From our analysis we obtain a $v \sin i$ of $7.2 \pm 0.15 \mathrm{~km} \mathrm{~s}^{-1}$, significantly lower from the value listed in the KIC catalogue. For Object- 1 the lower $v \sin i$ gives a low $\sin I_{\star}$ value $=0.27 \pm 0.08$, which in turns suggest that Object-1 has a significant spin-orbit mis-alignment. In Fig. 1-right panel, we show the Fourier transform of the LSD profile for Object-1. Object-2, is a G-type star with $T_{\text {eff }}$ of $5627 \mathrm{~K}$ hosting two planets a SuperEarth and a Neptune size planet. For Object-2 our analysis yields a $v \sin i$ of $\sim 1$ and thus we can not conclude on the spin-orbit orientation of this system. This is because of the degeneracy in the measurement of $I_{\star}$ along the line of sight. Thus the case of $I_{\star}=90^{\circ}$ (i.e., $\sin I_{\star}=1$ ) is analogous to the case of $\lambda=0^{\circ}$ for an RM measurement, which does not always imply a spinorbit alignment. Therefore statistical treatments are essential to compare the observed distributions

\footnotetext{
${ }^{1}$ IRAF is distributed by the National Optical Astronomy Observatory, which is operated by the Association of Universities for Research in Astronomy, Inc., under cooperative agreement with the National Science Foundation.
} 
with planetary migration theories. The SOAPS project will provide a much larger sample of systems with measured spin-orbit alignment, testing formation and evolution theories for different planetary architectures.

\section{References}

[1] Mayor, M., \& Queloz, D., 1995, Nature, 378, 355

[2] Lin, D. N. C., Bodenheimer, P., \& Richardson, D. C., 1996, Nature, 380, 606

[3] Rasio, F. A., \& Ford, E. B., 1996, Science, 274, 954

[4] Chatterjee, S., Ford, E. B., Matsumura, S., \& Rasio, F. A., 2008, ApJ, 686, 580

[5] Fabrycky \& Tremaine 2007, ApJ, 669, 1298

[6] Rossiter, 1924, ApJ, 60, 15

[7] McLaughlin, 1924, ApJ, 60, 22

[8] Hirano, T., Sanchis-Ojeda, R., Takeda, Y., et al., 2012, ApJ, 756, 66

[9] Gaudi \& Winn, 2007, ApJ, 655, 550

[10] Simpson, E. K., Pollacco, D., Cameron, A. C., et al., 2011, MNRAS, 414, 3023

[11] Winn, J. N., Fabrycky, D., Albrecht, S., \& Johnson, J. A., 2010, ApJL, 718, L145

[12] Schlaufman, K. C., 2010, ApJ, 719, 602

[13] Valenti, J. A., \& Fischer, D. A., 2005, ApJS, 159, 141

[14] Borucki, W. J., Koch, D. G., Basri, G., et al., 2011, ApJ, 736, 19

[15] McQuillan, A., Aigrain, S., \& Roberts, S., 2012, A\&A, 539, A137

[16] Batalha, N. M., Rowe, J. F., Bryson, S. T., et al., 2012, arXiv:1202.5852

[17] Gray, D. F., 1982, ApJ, 262, 682

[18] Dravins, D., Lindegren, L., \& Torkelsson, U., 1990, A\&A, 237, 137

[19] Kochukhov, O., Makaganiuk, V., \& Piskunov, N., 2010, A\&A, 524, A5

[20] Brown, T. M., Latham, D. W., Everett, M. E., \& Esquerdo, G. A., 2011, AJ, 142, 112

[21] Carney, B. W., Gray, D. F., Yong, D., et al., 2008, AJ, 135, 892 